Revista Mexicana de Economía y Finanzas, Nueva Época

Volumen 17 Número 2, Abril - Junio 2022, pp. 1-26, e609

DOI: https://doi.org/10.21919/remef.v17i2.609

(Recibido: 15/marzo/2021, aceptado: 23/agosto/2021, publicado: 26/octubre/2021)

\title{
El desempeño financiero de la empresa y la composición del consejo de administración
}

\author{
Adriana María Berrocal González ${ }^{1}$ - Deloitte, México \\ Lili Domínguez Ortíz ${ }^{2}$ - Universidad Iberoamericana, México \\ Fernando José Mariné Osorio ${ }^{3}$ - Universidad Anáhuac, México \\ Liliana Raquel Ruiz Fuentes ${ }^{4}$ - Universidad del Valle de México, México
}

Con base en la Teoría de Dependencia de Recursos (TDR) y la Teoría de Agencia (TA), esta investigación tiene por objetivo analizar si existe una relación entre el número y tipo de consejeros (constructo consejo de administración) con respecto a indicadores de desempeño financiero de la empresa con base en ROA, ROE, Margen de Beneficio Neto, ingresos por empleado y riesgo sistemático de mercado-beta (constructo desempeño financiero). Utilizando el método innovador y adecuado para muestras pequeñas PLS-SEM, se crea un modelo teórico basado en la revisión de literatura y un modelo de medición que opera con base en correlaciones múltiples y simultáneas entre las variables seleccionadas. Los resultados muestran la conformación de dos constructos y denotan una relación estadísticamente significativa entre ambos. Se tiene la limitante de ser un análisis exploratorio y transversal. El estudio es original en la aplicación del método estadístico al campo del gobierno corporativo y las finanzas y en la selección de variables. Se concluye que el modelo teórico planteado es eficiente para continuar estudiando los constructos en las empresas.

Clasificación JEL: C32, C51, G34, G38, G39, G30.

Palabras clave: consejo de administración, consejeros independientes, desempeño financiero de la empresa, PLS-SEM.

\section{Company's Financial Performance and Board of Directors Composition}

Based on the Resource Dependence Theory (TDR) and the Agency Theory (TA), this research aims to analyze whether there is a relationship between the number and type of directors (construct board of directors) concerning indicators of financial performance of the company based on ROA, ROE, Net Profit Margin, Income Per Employee, and Beta or Systematic Risk (construct financial performance). Using the innovative and suitable method for small samples, PLS-SEM, authors create a theoretical model based on literature review and a measurement model that operates based on multiple and simultaneous correlations between the selected variables. The results show the conformation of two constructs and denote a statistically significant relationship between them. It has the limitation of being an exploratory and transversal analysis. The study is original in applying the statistical method to the field of corporate governance and finance and in the selection of variables. It is concluded that the proposed theoretical model is efficient in studying the constructs in companies.

JEL Classification: C32, C51, G34, G38, G39, G30.

Keywords: board of directors, independent directors, company financial performance, PLS-SEM, corporate governance.

1 ORCID: https://orcid.org/0000-0001-9908-5259

2 ORCID: https://orcid.org/0000-0003-4265-5294

3 ORCID: https://orcid.org/0000-0002-9979-0430

${ }^{4}$ Autor de correspondencia. ORCID: https://orcid.org/0000-0002-9058-3865; Email: liliana.ruiz@uvmnet.edu

*Sin fuente de financiamiento para el desarrollo de la investigación 


\section{Introducción}

Existe cierto consenso entre investigadores de diversas latitudes respecto a la estrecha relación entre las prácticas de gobierno corporativo (GC) y el desempeño financiero en las empresas (Bhagat y Bolton, 2008; Fulghieri y Suominen, 2012; Ayda et al.,2017) y más aún, de la relación entre el desempeño financiero y la conformación del consejo en términos de tamaño e independencia (UribeBohorquez et al., 2018).

Uno de los elementos comúnmente estudiados en la relación de prácticas de GC con el desempeño financiero de la empresa es el tamaño y la independencia del consejo de administración (Brown y Caylor, 2004; Garg, 2007; Fuzi et al., 2016; Zabri et al., 2016; Alabdullah et al., 2018; Rashid, 2018; Zhou et al., 2018; Kao et al.,2019), siendo los resultados en su mayoría consistentes sobre la manera en que se relacionan estos constructos.

En la comunidad académica la base de datos Science Direct muestra un crecimiento sostenido del volumen de artículos año con año, en torno a este tema, desde inicios del siglo XXI. Si bien en los años más recientes, los estudios corresponden en buena medida a economías emergentes como India, China, Malasia y Turquía entre otras (Topak, 2011; Liu., 2015; Fuzi et al., 2016; Alabdullah et al., 2018), también se tiene el antecedente de estudios en economías desarrolladas algunos en países como España, Alemania, Reino Unido y Estados Unidos (Barnhart et al., 1994; Klein, 1998; Guest, 2009; Arosa., 2010, 2013; Bermig y Frick, 2010). Un tercer grupo de estudios se puede encontrar sobre países en Asia y Oceanía (Kim, 2005; Alabdullah et al., 2018; Rashid, 2018; Kao et al., 2019).

Con respecto a América Latina y en particular para México, se identifica un vacío en el estudio del tema y es en ello en lo que la presente investigación se propone contribuir (Baker et al., 2020; Watkins, 2018).

Estudios realizados por Chong y López de Silanes $(2006,2007)$ señalan la ausencia de un funcionamiento adecuado de los mercados de capitales en América Latina, ante un marco legal deficitario y la falta de mecanismos de control que promuevan el desarrollo de prácticas de GC. Dichos autores concluyen que en México existe evidencia de que más de una década de baja protección al inversionista ha tenido como consecuencia que el mercado de valores de este país sea uno de los más pequeños y con menores niveles de desarrollo en el mundo. Ya Macías y Román (2014) describían la necesidad de incidir en la protección al accionista minoritario para promover mejoras en las prácticas de GC en las empresas mexicanas; la relevancia de las buenas prácticas de gobierno corporativo en el desempeño financiero de la empresa en particular en México, se pone de manifiesto también en Valencia Herrera y Ruiz Rivera (2019) quienes encuentran que los inversionistas institucionales evitan las empresas con alto nivel de control familiar y optan en su lugar por el riesgo de mercado; los autores destacan una relación entre elementos de gobierno corporativo y la rentabilidad de las empresas.

En el contexto de esta corriente descrita de Law \& Finance 5 (Porta et al., 1998), la composición del consejo de administración es una de las variables más utilizadas para estudiar la relación entre prácticas de gobierno corporativo y el desempeño financiero de las empresas (Reyna, 2012). Conocer

\footnotetext{
${ }^{5}$ Se utiliza el anglicismo ya que hace referencia a una corriente de pensamiento que no hay otra forma de representar y no solo a un concepto o término.
} 
e impulsar de una mejor manera la conformación del consejo de administración puede contribuir a fortalecer el mercado. En la actualidad, ante la baja protección al inversionista y la tercera concentración de propiedad más alta en el mundo (con tasas de 67\%, tan sólo después de Grecia y de Colombia), las grandes empresas migran a mercados de valores en otras latitudes para tener acceso al capital, a veces con mejores términos, como lo demuestra el hecho de que el 38\% de las empresas listadas en el mercado mexicano estén también listadas en EE.UU (Chong y López de Silanes, 2007).

Al tenor de la literatura revisada, el objetivo de esta investigación es analizar si existe una relación estadística entre el número y tipo de consejeros con respecto a indicadores de desempeño financiero de la empresa. Para ello, se crea un modelo teórico basado en la revisión de literatura y para el tratamiento de datos empíricos, se utiliza un método especialmente apto para muestras pequeñas e innovador, de correlaciones múltiples y simultáneas, entre las variables seleccionadas que es el método PLS-SEM.

Esta investigación con base en la literatura, propone un modelo teórico y uno de medición, que permiten organizar la evidencia empírica del tamaño e independencia del consejo de administración y examinar el grado de correspondencia entre los resultados esperados que proporciona el modelo y las observaciones (Vargas Chanes, 2019). La investigación que a continuación se desarrolla, abona evidencia empírica para las empresas en México y contribuye al conocimiento teórico en el campo del GC.

Para este análisis se utilizó la información de las respuestas al "Cuestionario para Evaluar el Grado de Adhesión al Código de Principios y Mejores Prácticas de Gobierno Corporativo"6 (CCE, 2018 b), reportado anualmente por las empresas listadas en la BMV. El Código surgió en el marco de reformas que buscaban mejorar la rendición de cuentas a los inversionistas externos y teniendo como base el estudio de los cambios voluntarios y obligatorios de GC en las empresas mexicanas (Macías y Román, 2014). Por otra parte, para el desempeño financiero se utilizaron indicadores de tres categorías diversas: retorno empresarial, valor por empleado y riesgo de mercado, tomados de los reportes financieros anuales de las Empresas ${ }^{7}$.

El análisis teórico es conducido a la luz de dos teorías que fijan la mirada en el grado de control interno o externo que ejerce una organización, para gestionar las presiones del sistema empresarial en el que se desenvuelve y para alcanzar las metas deseadas, siendo éstas: a) la teoría de la dependencia de recursos (TDR) (Pfeffer y Salancik, 1978, 2003) que pone el acento en el control externo, la participación de consejeros independientes y la capacidad de adaptación de la organización a su entorno, y b) la teoría de agencia (TA) (Jensen y Mekling, 1976), relacionada con el control interno y los conflictos que puedan surgir entre el binomio principal-agente o principalprincipal.

Para alcanzar el objetivo planteado, la investigación se desarrolla en cuatro apartados. El primero presenta la revisión de literatura con un análisis dialógico del tema, incluyendo el modelo teórico propuesto con sus variables. El segundo apartado describe la metodología utilizada para examinar la relación entre el tamaño de consejo y los tipos de consejeros y variables de desempeño financiero de la empresa. Esta sección incluye las fuentes de información y los datos utilizados de 31

\footnotetext{
${ }^{6}$ Anexo 1 del Código de Principios y Mejores Prácticas de Gobierno Corporativo, emitido por el Consejo Coordinador Empresarial (CCE) en el año 2018.

${ }^{7}$ Consultados en Eikon Reuters en junio 2019 utilizando datos de enero-diciembre 2018.
} 
empresas $^{8}$ que conforman el IPC en 2019 en México. El tercer apartado presenta de forma detallada la naturaleza y los resultados del modelo teórico con base en la información empírica. Finalmente, el cuarto y último apartado expone las conclusiones y nuevas líneas de investigación identificadas.

\section{Estado del arte}

Si bien es cierto que el GC se define como un "sistema bajo el cual las sociedades son dirigidas y controladas" (CCE, 2018a) y tiene como objetivo principal, el desplegar acciones directivas para lograr la transparencia y rendición de cuentas que favorezcan las inversiones a largo plazo, la estabilidad financiera y la integridad en los negocios (OECD, 2016); un enfoque más amplio comprende el despliegue de un esquema de gobernanza que le garantice a la empresa estar bien dirigida y encaminada en la dirección correcta (Tricker, 2012; Ullah et al., 2018) .

Sin lugar a dudas, el GC cobra cada vez mayor importancia debido a su impacto en la generación de valor e ingresos en las organizaciones; ya que ha dejado de ser un instrumento exclusivo de protección de los accionistas minoritarios, para convertirse en una herramienta fundamental para disminuir los riesgos en las inversiones e incrementar la confianza de los accionistas con mayores dividendos y flujos libres de efectivo, que permitan mantener un crecimiento de largo plazo de la organización (Hernández y Berrocal, 2009; Zabri et al., 2016; Tosun, 2020).

Desde el enfoque teórico, la TDR-en seguimiento a los postulados seminales de Pfeffer y Salancik (1978; 2003)- aborda como punto central, el examinar la habilidad y efectividad con la que una organización adquiere y gestiona tanto recursos tangibles como intangibles del entorno siempre cambiante y demandante en el que se desenvuelve; mediante la operación de diversas y continuas interacciones con sus grupos de interés con quienes crea interdependencias que implican inevitablemente, procesos de adaptación y colaboración; más externos que internos y, sobre los cuales, debe ejercer un control activo para obtener los recursos de los que carece.

Esta teoría en relación con el GC, al exponer como punto medular la mirada externa de la organización, subraya la importancia de contar con un número amplio de miembros y de consejeros independientes en el consejo de administración (Hillman et al., 2009) que aporten experiencia, acceso a canales de información valiosa, legitimidad y una red amplia de contactos que les permita minimizar su dependencia externa de recursos, así como tomar decisiones estratégicas que reduzcan sus riesgos, especialmente en industrias altamente reguladas (Louma y Goodstein, 1999).

Por otra parte, la teoría ya clásica de agencia, originalmente postulada por Jensen y Mekling (1976) -la cual ha sido severamente criticada por Perrow (1993) debido a su debilidad para obtener por sí misma resultados empíricamente comprobables- alude a un enfoque interno de la organización respecto a la relación en la que una o más personas (el principal) contratan a otras personas (el agente) para realizar determinado servicio a su nombre; hecho que implica necesariamente un grado de delegación de autoridad del principal hacia el agente, quien no siempre actuará en beneficio del

8 El IPC de la BMV está compuesto generalmente por 35 empresas. Para el presente caso de análisis, no fue posible obtener de forma íntegra la información utilizada en el análisis de algunas empresas. Estas 31 empresas seleccionadas representan al 90\% el comportamiento del IPC de la BMV 
principal, sino que exhibirá una tendencia a favorecer sus propios objetivos, inclusive en algunos casos, a mostrar una actitud al riesgo opuesta a la del principal. En virtud de lo anterior, resulta imprescindible para el principal, establecer mecanismos contractuales de incentivos y de control, así como inevitable, el incurrir en ciertos costos transaccionales (Gompers et al., 2003) con el fin de regular la interacción de ambas partes para maximizar la utilidad o para evitar la erosión de valor de los recursos asignados a través de diferentes mecanismos (Dey, 2008). En este sentido, bajo los postulados de la TA, las organizaciones tenderán a estructurar un consejo de administración con un número reducido de miembros que puedan interactuar de forma muy cercana y frecuente con el principal, y afines al mismo.

Estas dos posturas teóricas resaltan la necesidad de mantener centrada la atención en la estructura y calidad de los sistemas estratégicos y de monitoreo del agente en la empresa, función que dentro del corpus de conocimiento del sistema de GC se encuentra asignada al consejo de administración como responsable de "definir el rumbo estratégico, vigilar la operación y aprobar la gestión de la dirección” (CCE, 2018b P. 11). Una de las mejores prácticas a este respecto, consiste en instalar un consejo de administración integrado por un número de consejeros que se encuentre en un rango de tres a quince miembros.

Efectivamente, el tamaño del consejo es un mecanismo de GC que parece estar identificado con la calidad de la gobernanza de la organización (Bermig y Frick, 2010; Topak, 2011; Arosa et al., 2013; Alabdullah et al., 2018) y por ende, con el desempeño financiero de la misma. Así lo vislumbraron estudios desarrollados en el ecosistema empresarial italiano (Di Pietra et al., 2008), al tratar de evaluar precisamente la relación existente entre el tamaño del consejo, el nivel de participación activa de sus consejeros en múltiples consejos y el valor accionario de la empresa en el mercado, durante un periodo de estudio de ocho años (1993-2000).

En este mismo sentido, estudios que involucraron 500 firmas listadas en regiones tan distantes cultural y económicamente de México como las asiáticas, confirman una relación positiva fuerte en la industria manufacturera de Vietnam entre mecanismos de GC -como son el tamaño y la independencia del consejo- y el desempeño financiero medido en términos del Retorno sobre el Capital (ROE), la Rentabilidad sobre Activos (ROA), el Retorno sobre Ventas (ROS), el crédito mercantil o "goodwill"9 y el retorno de los precios accionarios (Kabir y Minh Thai, 2017). Esta situación encuentra sintonía con los hallazgos de Wang et al., (2013) quienes examinaron más de 2,310 transacciones en las bases de datos de RiskMetrics y COMPUSTAT en el periodo de 1996 a 2006, llegando a la conclusión de que las grandes compañías, diversificadas y con alto apalancamiento tienden a conformar consejos de administración de gran tamaño, en tanto que compañías menos complejas, tienden a formar consejos más pequeños.

En el marco del análisis dialógico presentado con anterioridad, resaltamos los estudios teórico-empíricos realizados en economías emergentes como la colombiana por Orozco, Vargas y Galindo-Dorado (2018) quienes analizaron a la luz de la TA, la relación entre el tamaño del consejo y el desempeño financiero y reputacional de 84 compañías líderes en su giro en el periodo 2008-2012. El abordaje de estos autores gira en torno a la mejor práctica para Latinoamérica emitida por la Organización para la Cooperación y el Desarrollo Económicos (OECD) que señala como tamaño óptimo de este órgano de gobierno, un rango que varía entre cinco y nueve consejeros clave.

\footnotetext{
${ }^{9}$ Que en México se identifica como crédito mercantil
} 
En tal empeño, estos autores contrastan los supuestos inmersos en la TDR versus los razonamientos de la TA, afirmando que los consejos con más de siete miembros (consejos grandes) poseen una mejor reputación, pero un desempeño financiero más bajo; comparado con consejos de menos de siete miembros (consejos pequeños) que exhiben un mejor desempeño financiero. Esta situación converge con diversas investigaciones como la de Guest (2009), realizada sobre una muestra de 2,746 compañías británicas en el periodo de 1981-2002, que examina el impacto negativo de consejos grandes sobre la rentabilidad empresarial; la de Bhagat y Bolton (2008) que muestra una correlación negativa entre la independencia del consejo y el desempeño financiero de la empresa en el corto plazo, y las de estudios longitudinales realizados en 72 empresas provenientes de 12 sectores industriales, listadas en la bolsa de valores de Abu Dabi y Dubai en el periodo 2010-2013. Estos últimos revelan que en los Emiratos Árabes Unidos, donde el promedio de los consejos están compuestos por ocho miembros, los consejeros independientes no están motivados para servir al desempeño de la firma; situación que de acuerdo a los autores es explicable cuando sus directores externos participan en un gran número de consejos (Farhan, Normala Obaid y Azlan, 2017; Song et al., 2020).

Al respecto, es importante resaltar los supuestos de Jensen (2010) quien argumenta que el mantener un consejo con un número reducido de miembros, sin rebasar un numero ideal de siete a ocho, mejora el desempeño financiero interno de la organización, en virtud de la participación abierta y activa de los miembros del consejo, lo cual promueve invariablemente, una efectiva evaluación y monitoreo de la administración de la empresa.

En este sentido, resultados de investigaciones como la de Watkins (2018) en el mercado mexicano muestran que hay una diferencia significativa en el desempeño financiero de las empresas familiares con prácticas de GC, al considerar de forma particular el indicador ROA como la variable de desempeño. Encontrándose que tienen mejor desempeño las firmas con mayor concentración familiar en la tenencia accionaria y/o posiciones de liderazgo, debido a que, en las empresas familiares, las normas y reputación, pueden sustituir la falta de protección institucional formal para los grupos de interés que habitualmente poseen mediante mecanismos de GC. Los hallazgos de Watkins indican que el reducir la distancia entre el principal y el agente reduce los problemas y costos de agencia, y la eficacia de mecanismos de GC como la inclusión de consejeros independientes parecería diluirse.

Con respecto a las variables de GC, el análisis dialógico articulado previamente confirma que la participación de consejeros independientes -que impacta en el tamaño y la independencia del consejo- es uno de los elementos comúnmente analizados; más aún, los estudios teóricos-empíricos presentados permiten comprobar que, para medir el desempeño financiero de las empresas, las variables seleccionadas para este estudio son comúnmente utilizadas como en el caso de las variables de retorno.

Finalmente, cabe mencionar que en esta investigación se presentan tres elementos de aportación novedosa, uno es la conformación de un constructo de retorno empresarial (construido reflexivamente de acuerdo a la correlación presentada entre las variables) el segundo es la variable de medición de riesgo sistemático como es la beta (que también refleja correlación de los retornos accionarios al mercado) y los ingresos por empleado que proporciona una medición de la eficiencia empresarial al relacionar las ventas y el número de empleados. Un tercer elemento innovador es la 
modelación del objeto de estudio de esta investigación mediante el uso de mínimos cuadrados parciales y ecuaciones estructurales (PLS-SEM), cuyas ventajas de modelación se expondrán en siguientes secciones.

\section{Modelo teórico y operacionalización de variables}

A lo largo del tiempo se han utilizado métodos diversos para evaluar la realidad que emana de las prácticas de GC para atraer inversiones, maximizar sus recursos de capital y con ello mejorar el desempeño de la empresa y promover su crecimiento (Al-Matari et al., 2014).

La complejidad de medir el desempeño de la empresa reside en determinar de forma explícita las variables que conforman el constructo social de desempeño financiero (Neely et al., 1995) y lo que una investigación, como la que aquí se presenta, analiza en un contexto empresarial determinado (Lebas y Euske, 2002). La figura 1 muestra el abordaje teórico propuesto en la presente investigación, con base en la revisión de literatura descrita en el apartado anterior.

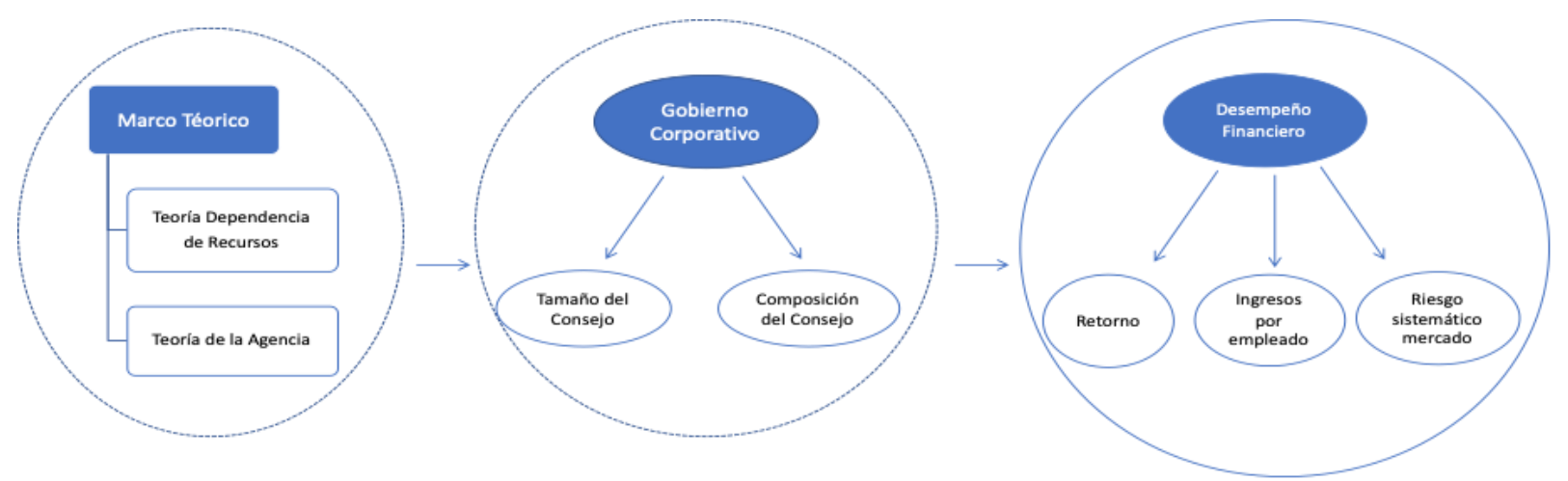

Figura 1. Modelo teórico para el análisis de la relación entre mejores prácticas de Gobierno Corporativo y el desempeño financiero de la empresa.

Fuente: Elaboración propia.

\subsection{Variables seleccionadas}

Con base en la revisión de literatura para el análisis en la presente investigación, se han seleccionado indicadores financieros, desde las ópticas de rentabilidad con indicadores que suelen ser utilizados por el grupo inversor como los de retorno empresarial (ROA, ROE y Net Profit Margin - margen de beneficio neto), y otros menos comúnmente utilizados como el riesgo sistemático (Beta), y los ingresos por empleado. La conceptualización y operacionalización de variables se describe en la tabla 1.

En cuanto a los indicadores tomados de los reportes financieros anuales de las empresas, describimos en primera instancia, la selección de la variable Beta de mercado, la cual presenta la relación lineal entre el excedente de margen del mercado sobre la tasa libre de riesgo y el activo analizado. Este indicador ha sido ampliamente utilizado en el mundo financiero moderno como medida de la sensibilidad (elasticidad) del retorno de un activo financiero ante cambios en indicadores de mercado, cuya naturaleza puede ser bursátil (Sharpe, 1964; Lintner, 1965), o bien de 
otro tipo y a la vez multifactorial, como lo expusieron Ross (1976) y Fama y French (1993; 2105). Su importancia en la literatura financiera radica en que es comúnmente utilizada en la aplicación del Modelo de Valoración del Precio de los Activos Financieros o Capital Asset Pricing Model (conocido como CAPM) y la determinación del premio al riesgo que el inversionista está dispuesto a aceptar por llevar a cabo una inversión en determinado activo. Por ende, también se le conoce como el componente del riesgo sistemático -en el que se considera el sistema como algo exógeno. Black (2001) sostiene al analizar una muestra de 21 firmas rusas del año 1999- que el comportamiento corporativo afecta el valor de mercado, lo que nos lleva a pensar que dicha relación puede ser significativa en el presente estudio.

Por otra parte, existe extensa literatura sobre indicadores de retorno empresarial como lo son el ROA, ROE y Margen de Beneficio Neto. De acuerdo con Reuters, el ROA es una relación de rentabilidad que mide el rendimiento de una compañía en relación con el total de sus activos. El ROA se mide sin tener en cuenta el apalancamiento que utiliza una empresa. Se calcula dividiendo el ingreso neto (net income) de una empresa antes de los costos de financiamiento entre los activos totales ${ }^{10}$. Así también, el ROE es la rentabilidad sobre los recursos propios o el capital accionario: un índice de rentabilidad calculado, dividiendo el ingreso neto de una compañía entre el patrimonio total de las acciones comunes. Otro indicador analizado es el Margen de Beneficio Neto que es la utilidad neta dividida entre las ventas, ratio que expresa con qué porcentaje de utilidad se queda la empresa después de haber pagado impuestos, intereses, depreciación y amortización.

Por último, es relevante también seleccionar un criterio de eficiencia empresarial como es los ingresos por empleado, siendo éste, el cociente del total de las ventas entre el número de empleados. Es relevante comentar que el presente indicador conlleva la limitante particular de acuerdo con que dichas ventas pueden ser determinadas por el año, sector, industria específica, así como el número de empleados de acuerdo con las necesidades de cada giro o industria.

Tabla 1. Conceptualización y operacionalización de variables

\begin{tabular}{|l|l|l|}
\hline $\begin{array}{c}\text { Variables de } \\
\text { Gobierno } \\
\text { Corporativo }\end{array}$ & \multicolumn{1}{|c|}{ Conceptualización } & \multicolumn{1}{c|}{ Operacionalización } \\
\hline $\begin{array}{l}\text { Tamaño del Consejo } \\
\text { de Administración }\end{array}$ & $\begin{array}{l}\text { Número total de personas que forman el Consejo } \\
\text { de Administración en relación con las mejores } \\
\text { prácticas nacionales e internacionales de GC. }\end{array}$ & $\begin{array}{l}\text { Número total de personas que } \\
\text { forman el Consejo. }\end{array}$ \\
\hline $\begin{array}{l}\text { Consejeros } \\
\text { Independientes }\end{array}$ & $\begin{array}{l}\text { Consejeros que no están vinculados con el equipo } \\
\text { de dirección de la sociedad, no están sujetos a a } \\
\text { intereses personales, patrimoniales o económicos } \\
\text { y son llamados a formar parte del Consejo de } \\
\text { Administración por su experiencia, capacidad y } \\
\text { prestigio profesional. }\end{array}$ & \multicolumn{2}{|c|}{ consejeros } \\
\hline $\begin{array}{l}\text { Consejeros } \\
\text { Patrimoniales }\end{array}$ & $\begin{array}{l}\text { Consejeros accionistas que no forman parte del } \\
\text { equipo de dirección. }\end{array}$ & $\begin{array}{l}\text { Número de consejeros } \\
\text { patrimoniales. }\end{array}$ \\
\hline
\end{tabular}

10 La definición común de ingreso neto considera los ingresos después del pago de intereses; sin embargo, en este caso el dato utilizado es el de Eikon y de ahí se toma la definición que indica al ingreso neto sin considerar apalancamiento. 


\begin{tabular}{|l|l|l|}
\hline $\begin{array}{l}\text { Consejeros } \\
\text { Relacionados }\end{array}$ & Consejeros funcionarios de la sociedad. & $\begin{array}{l}\text { Número de consejeros } \\
\text { relacionados. }\end{array}$ \\
\hline $\begin{array}{l}\text { Variables de } \\
\text { Desempeño } \\
\text { Financiero }\end{array}$ & Conceptualización & Operacionalización \\
\hline $\begin{array}{l}\text { Rentabilidad sobre } \\
\text { el capital (ROE) }\end{array}$ & Es el rendimiento sobre el capital accionario. & $\begin{array}{l}\text { Se calcula dividiendo Ingreso } \\
\text { neto / Capital Accionario. }\end{array}$ \\
\hline $\begin{array}{l}\text { Rentabilidad sobre } \\
\text { activos (ROA) }\end{array}$ & Es el rendimiento sobre los activos empresariales. & $\begin{array}{l}\text { Se calcula dividiendo Ingreso } \\
\text { neto sin considerar } \\
\text { apalancamiento / Activos } \\
\text { totales promedio. (ver nota al } \\
\text { pie 8) }\end{array}$ \\
\hline $\begin{array}{l}\text { Margen } \\
\text { beneficio neto de }\end{array}$ & $\begin{array}{l}\text { Entendido como el porcentaje de utilidad neta en } \\
\text { relación con las ventas de la compañ́a. }\end{array}$ & $\begin{array}{l}\text { Se calcula dividiendo la } \\
\text { utilidad neta entre las ventas. }\end{array}$ \\
\hline $\begin{array}{l}\text { Beta } \\
\text { Entendido como un indicador del riesgo } \\
\text { sistemático de una acción o portafolio de acciones } \\
\text { con respecto al mercado en su conjunto. }\end{array}$ & $\begin{array}{l}\text { Regresión por mínos } \\
\text { cuadrados del indicador de } \\
\text { mercado versus la acción. }\end{array}$ \\
\hline $\begin{array}{l}\text { Ingresos } \\
\text { empleado }\end{array}$ & $\begin{array}{l}\text { Se calcula dividiendo las } \\
\text { ventas entre el número de } \\
\text { empleados. }\end{array}$ \\
\hline
\end{tabular}

Fuente: Elaboración propia

\section{Metodología}

Existen diversas metodologías para el estudio de efectos correlacionales entre múltiples variables. La mayoría de las metodologías utilizadas en las finanzas, tienen que ver con los estimadores de mínimos cuadrados ordinarios (OLS), particularmente la regresión lineal multivariada que es una técnica del siglo XVIII utilizada por Gauss antes de 1794 (López de Prado, 2018).

En la actualidad se cuenta con otro tipo de métodos estadísticos como lo son las ecuaciones estructurales SEM (Structural Equation Models) que pueden ayudar a realizar nuevas aproximaciones; éstos son denominados de segunda generación. Con estos métodos es posible generar constructos y abstraer la información de la que se dispone (Bagozzi, 1981); así como también gozar de flexibilidad en cuanto a la naturaleza de la investigación, ya sea exploratoria o confirmatoria. Los métodos de ecuaciones estructurales pueden ser basados en mínimos cuadrados parciales (PLSSEM) o basados en covarianza (CB-SEM) son de utilidad, si el objetivo es analizar múltiples correlaciones, contrastar diversas hipótesis y por ende estudiar, efectos sobre variables endógenas. Estos métodos permiten modelar supuestos que en los clásicos OLS no es posible.

PLS -SEM es una técnica estadística para series de estimaciones de ecuaciones simultáneas mediante regresiones múltiples y se caracteriza por dos componentes básicos: 1) el modelo estructural y 2) el modelo de medida" (Martínez Ávila y Fierro Moreno, 2018. p: 1). Por otra parte, los métodos PLS-SEM estiman los coeficientes que maximizan la R cuadrada, o bien los valores de los constructos endógenos, y no tanto la discrepancia entre covarianza muestral y estimada (Hair et al., 2014). En ese sentido los métodos SEM son de utilidad para generar inferencia causal (Valencia 
Herrera y Ruiz Rivera, 2019). Ciertamente la técnica de PLS- SEM puede ser usada tanto para la investigación explicativa (confirmatoria) como para la predictiva (exploratoria) y permite examinar simultáneamente una serie de relaciones de dependencia entre variables independientes y dependientes así como probar teóricamente modelos causales (Martínez Ávila y Fierro Moreno, 2018).

Reinartz et al.,(2009) muestran que la aplicación de métodos PLS-SEM logra una mayor convergencia en casos de muestras pequeñas, al ser comparado con métodos análogos basados en la covarianza.

Adicionalmente, Henseler et al., (2013) aseveran que en contraste con métodos CB-SEM, los métodos PLS-SEM pueden ser usados aun cuando el número de observaciones sea menor que el número de variables, sean éstas manifiestas o latentes. Así, ellos concluyen que, los métodos PLS-SEM pueden ser aplicados en muchos casos de muestras pequeñas donde otros métodos fallan. Una de las diferencias importantes entre los métodos de ecuaciones estructurales mencionados, es que la estimación con PLS-SEM se realiza por medio del método de mínimos cuadrados parciales mientras que la estimación con CB-SEM utiliza el método basado en el logaritmo de máxima verosimilitud.

Por lo tanto, el método utilizado en la presente investigación ofrece otra bondad en lo relativo al supuesto de distribución normal, ya que en los mercados financieros se observan regularmente problemas de falta de normalidad con la distribución de los errores, y éste no hace supuestos sobre la distribución de las observaciones. No obstante, una de las aportaciones importantes es que ha sido poco utilizado en estudios financieros (Avkiran y Ringle, 2018) y específicamente en el mercado mexicano (Mariné, 2019).

\subsection{Las Fuentes y los Datos}

La muestra utilizada es de corte transversal y está compuesta por 31 empresas del IPC. Estas

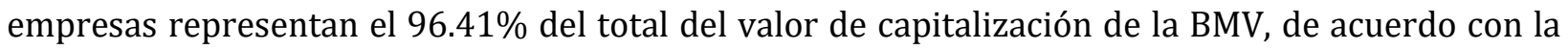
información obtenida de la plataforma Eikon Reuters, fuente de la información financiera analizada, correspondiente al periodo enero a diciembre de 2018.

La información relativa a las variables relacionadas con las prácticas de GC corresponde a los datos de las Empresas para 2018, reportados en el primer semestre de 2019.

Así y en el entendido de los datos disponibles, se procede a establecer la presente hipótesis.

\section{H1: El número de consejeros y la naturaleza de éstos, es significativo para ofrecer explicación causal del constructo desempeño financiero de la empresa, conformado por retorno empresarial, riesgo sistemático e ingresos por empleado.}

Esta hipótesis nos permite examinar si el tamaño y la composición del consejo de administración tiene un impacto significativo en el desempeño financiero de las Empresas durante el ejercicio fiscal de 2018 y arroja evidencia empírica para concluir su alineación, ya sea con los supuestos de la TDR o la TA. 


\section{Modelado y Resultados}

Para el estudio en cuestión se realizaron dos modelos. El primero de ellos es de naturaleza reflexiva en su totalidad y el segundo es de naturaleza formativa-reflexiva. Esta naturaleza en relevante ya que justamente una de las virtudes del método utilizado es que se puede utilizar con constructos formativos y reflexivos (Avkiran y Ringle, 2018). Con respecto al constructo desempeño financiero, se conforma por un grupo de variables de retorno empresarial (reflejado por ROA, ROE y Margen de Beneficio Neto), y una de riesgo sistemático (beta) y una de eficiencia en términos de ingresos por empleado (ventas por empleado).

Por otra parte, se utilizaron tres variables exógenas únicas, como lo son número de consejeros independientes, número de consejeros patrimoniales y número de consejeros relacionados, en tanto que los constructos financieros fueron tratados endógenamente como se puede observar en la figura 2 .

Para llegar al modelo de medición que se utiliza en este trabajo, se buscó en todo momento un modelo parsimonioso en el entendido de que puedan existir siempre variables adicionales que afecten el desempeño financiero de la empresa.

En cuanto al modelo de medición y los datos empíricos, el método PLS-SEM estima los coeficientes que maximizan la R cuadrada (Hair et al., 2014). Los métodos de ecuaciones estructurales (PLS-SEM) y los métodos de ecuaciones estructurales basados en covarianza (CB-SEM) son de utilidad, si el objetivo es analizar múltiples correlaciones de manera simultánea, contrastar diversas hipótesis y por ende estudiar, efectos sobre variables endógenas. (Hair et al., 2014).

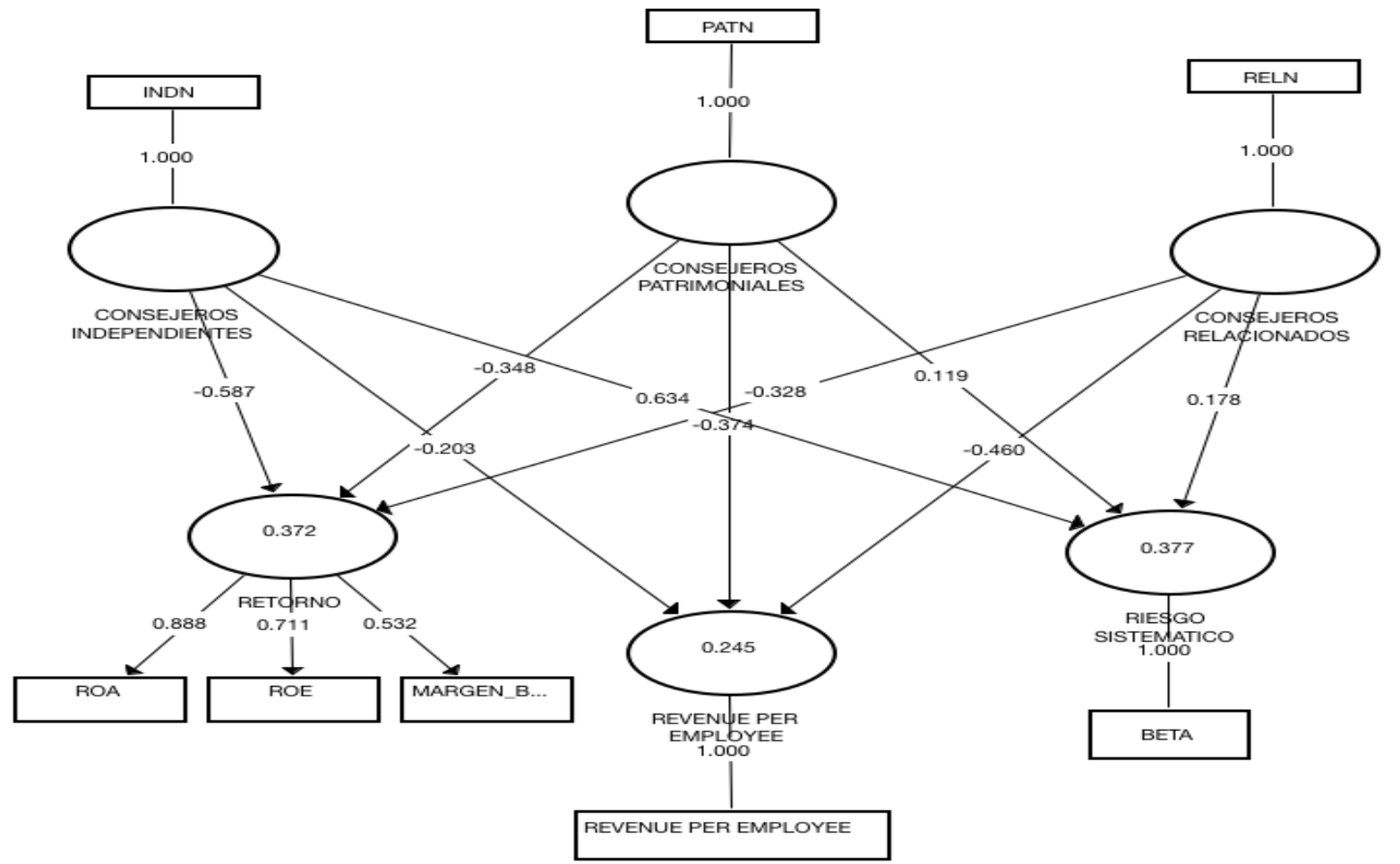

Figura 2. Path Analysis: Modelo reflexivo consejeros independientes, patrimoniales y relacionados. Fuente: Elaboración propia. 
La Figura 2 presenta el análisis con los estadísticos R-cuadrada que explican la varianza de los constructos endógenos de las regresiones parciales efectuadas. En concordancia con el número de datos, se esperaban R-cuadradas moderadas y bajas, ya que este indicador es el cociente entre la suma al cuadrado del modelo explicado entre la suma al cuadrado del modelo total. Así, se tienen Rcuadradas entre un rango (bajo-medio) del .24 a .37, siendo las mayores las relativas al constructo riesgo sistemático y retorno empresarial y, por otra parte, las menores se encuentran en el constructo de ingresos por empleado, como se aprecia en la tabla 2

Tabla 2. Path Analysis: Estadísticos Modelo reflexivo consejeros independientes, patrimoniales y relacionados

\begin{tabular}{|c|c|c|c|}
\hline Consejeros & $\begin{array}{c}\text { Retorno } \\
\text { empresarial }\end{array}$ & $\begin{array}{c}\text { Revenue per } \\
\text { employee }\end{array}$ & Riesgo sistemático \\
\hline INDEPENDIENTES & -0.587 & -0.203 & 0.634 \\
\hline PATRIMONIALES & -0.348 & -0.374 & 0.119 \\
\hline RELACIONADOS & -0.328 & -0.46 & 0.178 \\
\hline
\end{tabular}

Fuente: Elaboración propia

$\mathrm{Al}$ analizar los paths o senderos que delimitan las variables exógenas de las endógenas se observa en la tabla 2 en primera instancia que existe una relación negativa entre el número de consejeros independientes y dos constructos analizados: retorno empresarial e ingresos por empleado; siendo la de mayor relevancia predictiva de éstas con respecto al primero. Por otro lado, se presenta una relación positiva al riesgo sistemático o beta del mercado denotando también una relación fuerte predictiva.

Con relación al número de consejeros patrimoniales, se observa una asimetría en la relación entre las variables al presentar un signo negativo entre el retorno empresarial e ingresos por empleados; lo cual se aprecia en la tabla dos. Es decir, el número de consejeros patrimoniales también presenta una incidencia negativa salvo el caso del riesgo de mercado. Se puede observar también una relación negativa entre el número de consejeros relacionados y los constructos retorno empresarial e ingresos por empleado; por último se observa una relación positiva al constructo de riesgo sistemático.

En lo relativo a magnitudes, el efecto negativo de mayor magnitud se encuentra entre el número de consejeros independientes y el constructo retorno empresarial, así también, la mayor magnitud en sentido positivo se encuentra entre el constructo consejeros independientes y el constructo riesgo sistemático.

En la tabla 3, es posible apreciar la significatividad estadística de los parámetros calculados en el modelo. Para ello, se estructuraron pruebas de bootstrapping de 10,000 iteraciones para analizar los paths calculados, observando que, de acuerdo con los valores $t$ statistics y los $P$ values calculados, existe evidencia estadística entre el número de consejeros independientes y todos los constructos, salvo el caso de revenue per employee (0.112). En el caso de los consejeros patrimoniales se genera una relevancia significativa con todas las variables endógenas, salvo el caso de riesgo sistemático (0.480). Y para el caso de los consejeros relacionados, existe relevancia 
significativa con todos los constructos salvo con el constructo riesgo sistemático (0.128). Cabe señalar que, existe significatividad de todas las variables exógenas con el constructo retorno.

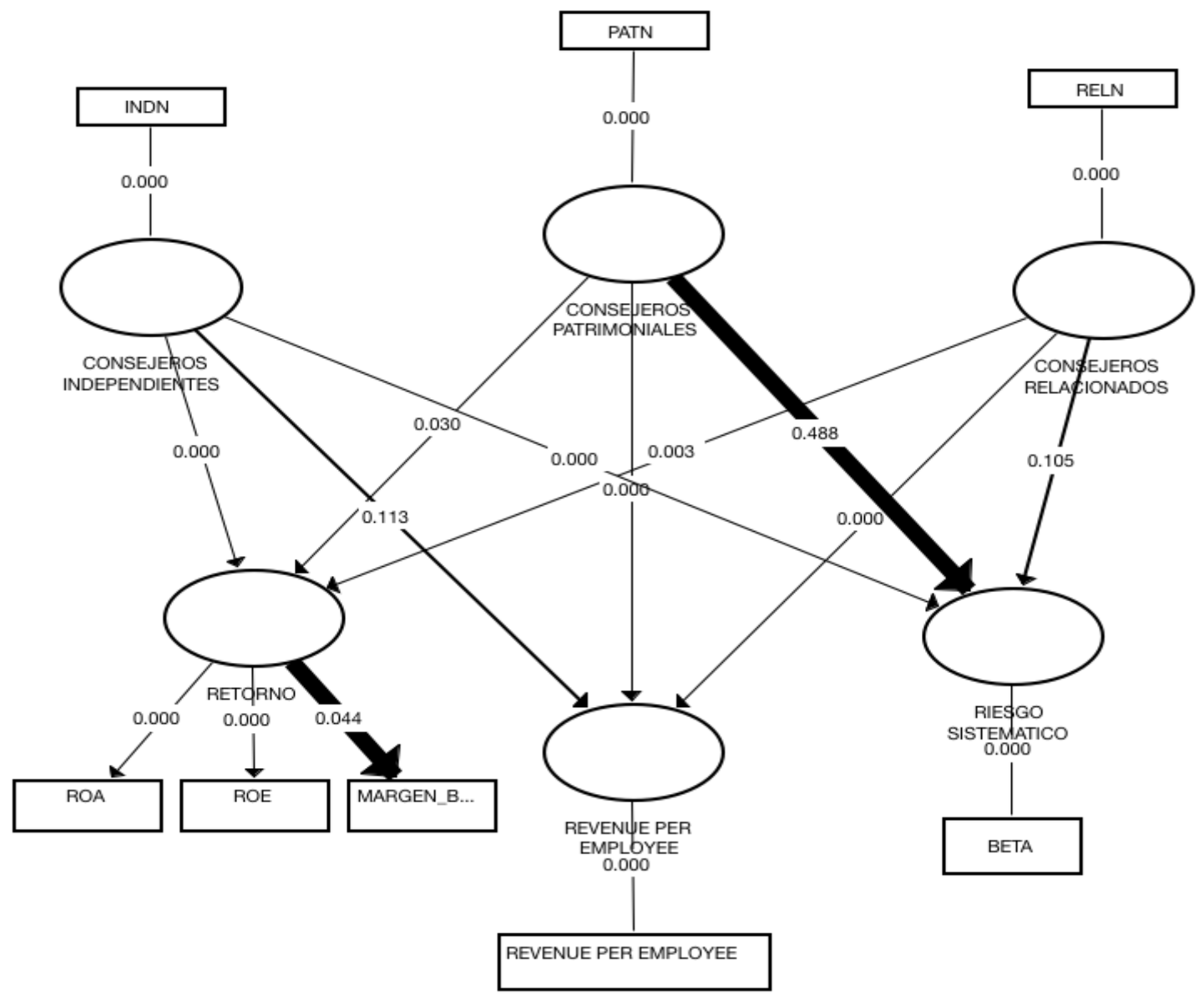

Figura 3. P-values: Modelo reflexivo consejeros independientes, patrimoniales y relacionados. Fuente: Elaboración propia

Se expone a continuación la validación de este primer modelo en seguimiento a Hair et al., (2019) y los indicadores que dichos autores recomiendan para la validación de la presente metodología.

Tabla 3. Validación del Modelo Reflexivo

\begin{tabular}{|l|l|l|}
\hline \multicolumn{1}{|c|}{ Modelo Reflexivo } & \multicolumn{1}{|c|}{ Criterios } & \multicolumn{1}{c|}{ Valuación } \\
\hline Loadings & Mayores a .7 & $\begin{array}{l}\text { Cumple. Para caso específico de Margen de } \\
\text { Beneficio Neto se encuentra bajo. Se incluye } \\
\text { esta variable bajo el criterio de AVE y debido } \\
\text { a que existe pocos indicadores para la } \\
\text { formación de dicho constructo. }\end{array}$ \\
\hline Composite Reliability & Mayor a .7 & Cumple \\
\hline Convergence Validity & AVE mayor a .5 & Cumple \\
\hline Discriminant Validity & Menor a .85 & $\begin{array}{l}\text { Cumple heterotrait-monotrait ratio of } \\
\text { correlations }\end{array}$ \\
\hline
\end{tabular}


REMEF (The Mexican Journal of Economics and Finance)

El desempeño financiero de la empresa y la composición del consejo de administración

\begin{tabular}{|l|l|l|}
\hline Colinearidad & Menor a 3 & Cumple VIF \\
\hline R cuadrada & Mayor a .25 a .50 & Efectos débiles a medios \\
\hline Q cuadrado & $\begin{array}{l}\text { Valores mayores a cero y 0 a .25 } \\
\text { son pequeña relevancia } \\
\text { predictiva del modelo }\end{array}$ & $\begin{array}{l}\text { Cumple criterio mayor a cero, con una } \\
\text { relevancia predictiva pequeña entre 0 y.25. }\end{array}$ \\
\hline PLS Predict & $\begin{array}{l}\text { Q<0 el modelo no se comporta } \\
\text { mejor que un modelo naive, se } \\
\text { compara versus un modelo tipo } \\
\text { LM para ver si PLS se comporta } \\
\text { mejor }\end{array}$ & $\begin{array}{l}\text { Para el caso de PLS no tiene ningún } \\
\text { problema el modelo. El modelo tiene poder } \\
\text { predictivo al generar ventajas de menor } \\
\text { error al comparar PLS- LM logrando } \\
\text { ventajas en RMSE, MAE y MAPE }\end{array}$ \\
\hline $\begin{array}{l}\text { Comparativas } \\
\text { modelos }\end{array}$ & Modelo minimiza BIC o GM & $\begin{array}{l}\text { Se buscó el menor indicador posible y se } \\
\text { trabajó con un modelo parsimonioso. }\end{array}$ \\
\hline
\end{tabular}

Fuente: Elaboración propia basado en Hair, Risher, Sarstedt y Ringle (2019).

De igual forma se elaboró un segundo modelo de naturaleza formativa-reflexiva construido formativamente sobre el constructo exógeno denominado número de consejeros, para su posterior análisis reflexivo sobre los mismos constructos endógenos estudiados previamente.

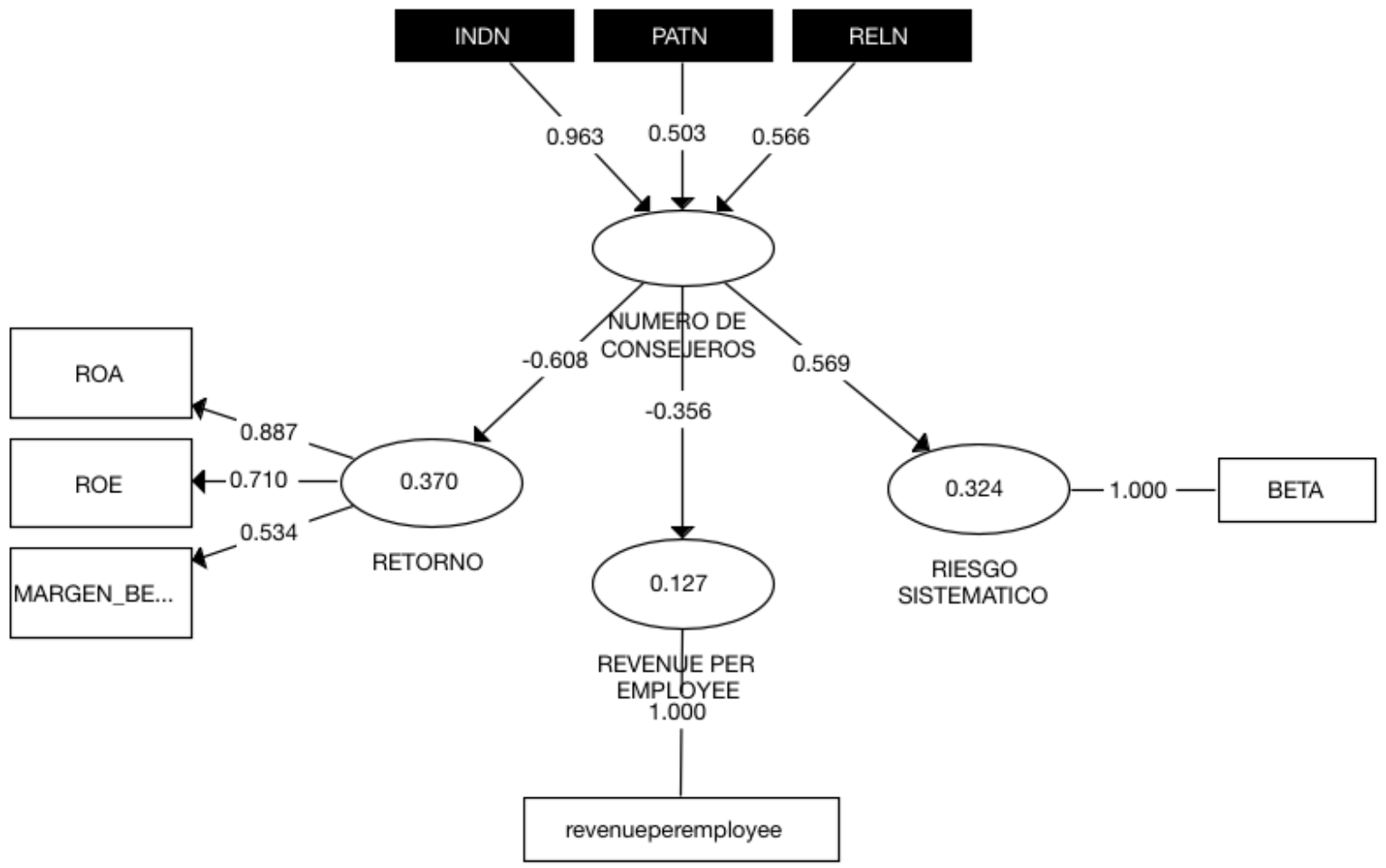

Figura 4. Modelo formativo-reflexivo consejeros independientes, patrimoniales y relacionados.

Fuente: Elaboración propia. 
Del presente modelo examinado en la figura 4 se puede cotejar que el mayor peso del constructo formativo denominado número de consejeros, lo aporta el número de consejeros independientes (.963) seguido de los consejeros relacionados (.566) y por último de los consejeros patrimoniales (.503) cuyo valor se encuentra justo en el límite para la aceptación de éste. El presente análisis ratifica la naturaleza de las relaciones entre los constructos -aunque se cuenta con una muestra pequeña- y evidencia también, una relación negativa entre indicadores internos de la empresa como lo son retorno e ingresos por empleado. Un hecho importante a destacar es la relación positiva a la beta de mercado representada por el constructo riesgo sistemático y por el número y tipo de consejeros analizados.

Asimismo, se realizaron pruebas sobre el constructo absoluto (ver anexo) resultando en un path de .83 lo que afirma el modelo en cuanto a su convergencia y su validez, bajo un criterio de 7 de acuerdo con Cheah et al., (2018).

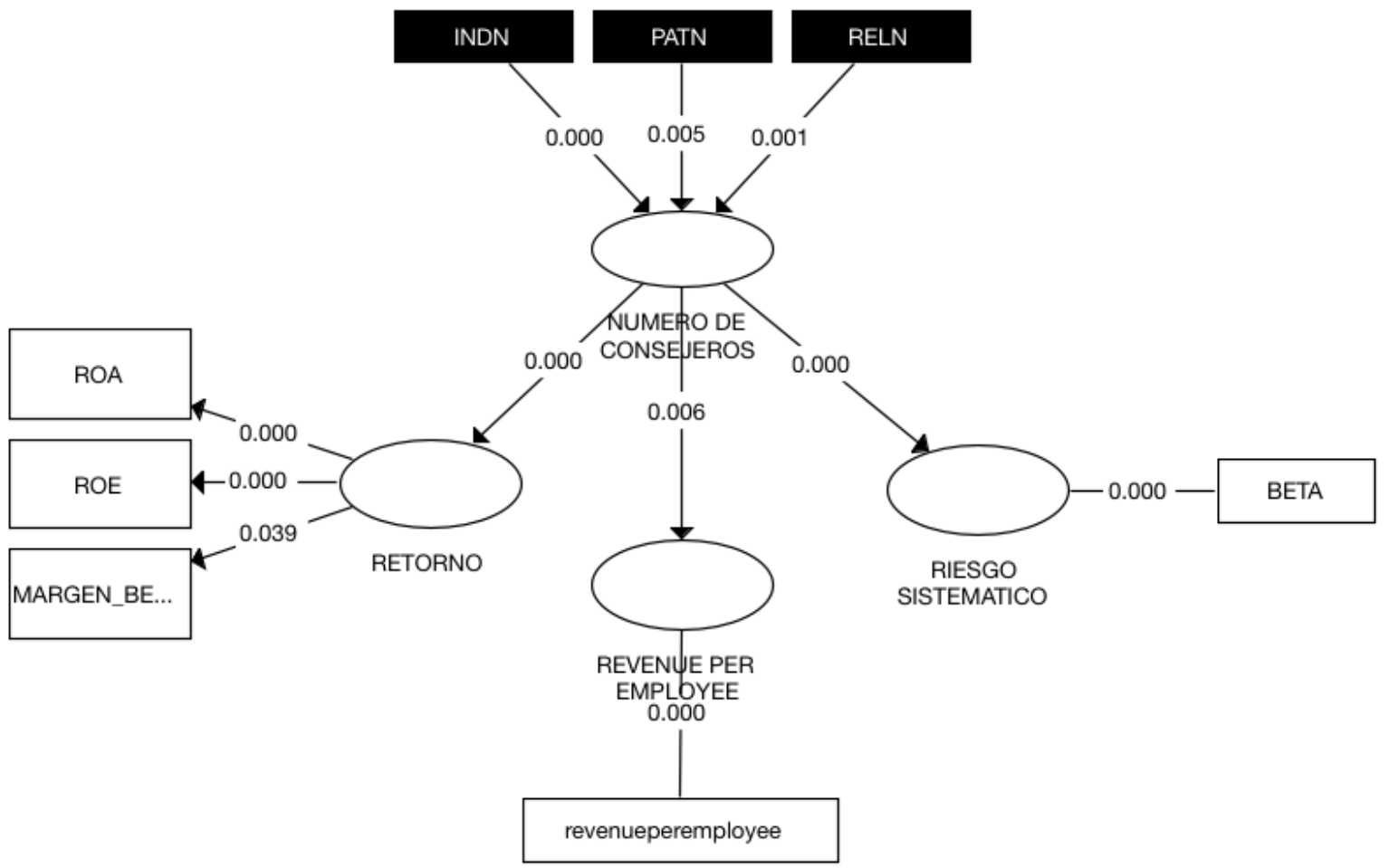

Figura 5. $P$-values Modelo formativo-reflexivo consejeros independientes, patrimoniales y relacionados.

Fuente: laboración propia.

Al analizar la significatividad del presente modelo por medio de la técnica de bootsrapping se muestra en la figura 5 que todos los constructos fueron significativos. En la perspectiva del constructo formativo-reflexivo del número de consejeros, el análisis denota la contribución relativa considerando todos los demás indicadores de los diversos tipos de consejeros; por ende, es posible afirmar que la mayor contribución relativa al constructo denominado "Número de consejeros" se encuentra en los consejeros independientes (Hair, Hult, Ringle y Saerstedt, 2014). 


\section{Conclusiones}

Tal y como lo confirman los datos, existe evidencia de la relación entre el número y tipo de consejeros y el constructo de desempeño financiero formado con información de 31 empresas que conforman el IPC del mercado mexicano de valores. Dicha relación es asimétrica en dos de los casos evaluados al ser modelada bajo PLS-SEM específicamente. Es así como bajo un supuesto de linealidad y dada la muestra utilizada y los constructos analizados, entre mayor número de consejeros, sin importar su naturaleza y tipo, se pueden esperar efectos negativos sobre los constructos retorno empresarial e ingresos por empleado. Este hecho converge con Orozco, Vargas, y Galindo-Dorado (2018) que estudian los consejos grandes y señalan que más de siete miembros otorgan una mejor reputación a la empresa, pero un desempeño financiero más bajo. Hecho también consistente con Dal Vesco y Beuren (2016) y en sintonía con las investigaciones de Yermack (1996) y Cheng (2008) que proveen evidencia empírica de que compañías con un gran consejo directivo o un número grande de consejeros, está asociado negativamente con la variabilidad de los rendimientos.

Sin embargo, este hecho es contrario en lo relativo al riesgo sistemático, es decir, al mercado y la relación de éste, en torno a los rendimientos de los precios empresariales. Es así como, en el caso específico del IPC de la Bolsa Mexicana de Valores, la variable exógena consejeros independientes presenta una correlación positiva y significativa a la beta del mercado como lo describe Saif Ullah (2012) quien afirma que existe una relación positiva entre la independencia del consejo directivo y los valores accionarios de la empresa, coincidiendo con aportaciones como las de Di Pietra et al., (2008) y Kabir y Minh Thai (2017). Para el caso de México, los resultados coinciden con Watkins, 2018 y con Valencia Herrera y Ruiz Rivera, 2019.

Otro hecho interesante, reside en el número de consejeros patrimoniales y relacionados que no presentaron significatividad estadística con relación al riesgo sistemático (modelo reflexivo) en contraste con el número de independientes que sí lo presentaron en la muestra analizada. Hecho que de acuerdo a la facultad predictiva-exploratoria del método PLS-SEM fue interesante de poder estudiar tanto reflexiva como formativamente y ello nos motiva a examinar en futuras líneas de investigación en dicho tema. Por otra parte, resulta interesante que al ser evaluado el número de consejeros como un constructo exógeno (modelo formativo-reflexivo) todas las variables tengan significatividad estadística con los diversos constructos, validando las correlaciones presentadas y la relevancia específicamente de los consejeros independientes.

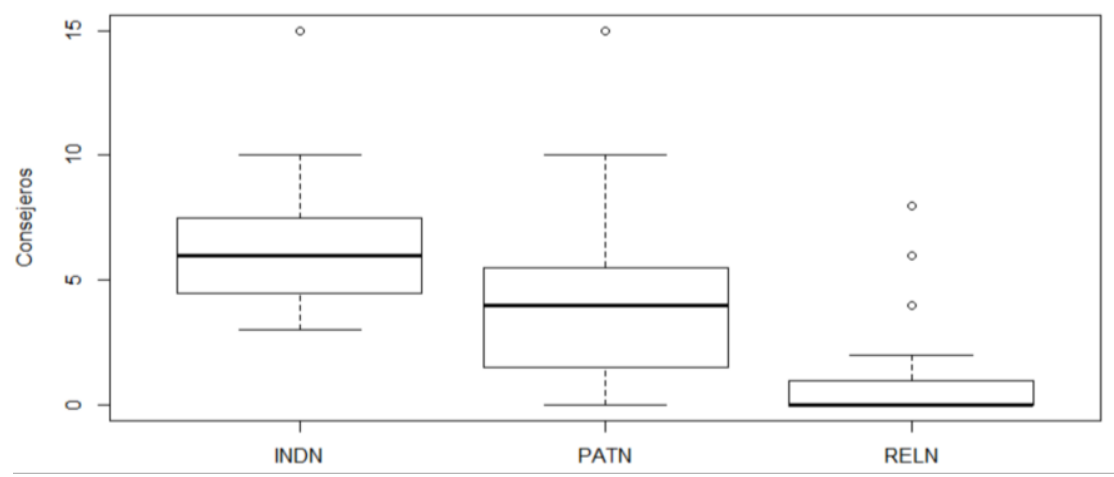

Figura 6. Estadística de los consejeros independientes, patrimoniales y relacionados. Fuente: Elaboración propia. 
Por otra parte, al analizar la estadística básica de las Empresas (figura 6), se observa que el número promedio de consejeros independientes es cercano a 6 , en tanto que el de patrimoniales es cercano a 4, y los relacionados cercano a uno. Las Empresas muestran un máximo de 15 consejeros para el caso de patrimoniales e independientes, y un máximo de 8 en consejeros relacionados.

Con respecto al método, mucho está por hacerse en el presente camino, y en lo relativo a comparativos sobre los sectores empresariales. Así, por ejemplo, resulta una limitante el que el estudio agrupa empresas de diversos sectores y es bien sabido que existen diferencias al respecto especialmente en las variables de desempeño financiero. Sin embargo, es importante hacer una pausa sobre las limitantes relativas a la micronumerosidad y la linealidad de los supuestos del modelado. Al evaluar la naturaleza del método se observan virtudes para la plasticidad de éste y menor error al ser comparado por algoritmos PLS-SEM, que se apoyan en técnicas como lo son bootstrapping, blindfolding y otros algoritmos de Machine Learning para la validación predictiva del presente método, tanto dentro como fuera de la muestra.

Debe comentarse que la presente investigación buscó en todo momento la parsimonia y entender las limitantes que preceden a todo estudio como lo son las limitantes de tamaño de muestra, año especifico estudiado, supuesto estadísticos y limitantes que puedan presentarse en épocas con características específicas en donde las relaciones entre variables pudieran ser modificadas por la naturaleza de la incertidumbre. Si bien, la estadística nos ayuda a clarificar el camino, mucho está por hacerse y estudiarse en futuras líneas de investigación.

Es así como el actual entorno competitivo, interconectado y con gran diversidad de riesgos, impulsa a las compañías más relevantes de la Bolsa Mexicana de Valores hacia postulados que se rigen por la TDR que apuesta en mayor medida por atender los factores externos, destacando la participación de un número mayor de consejeros independientes en el consejo de administración.

\section{Referencias}

[1] Alali, F., Anandarajan, A., \& Jiang, W. (2012). The effect of corporate governance on firm's credit ratings: further evidence using governance score in the Unitade States. Accounting \& Finance, 52, 291-312. https://doi.org/10.1111/j.1467-629X.2010.00396.x

[2] Alabdullah, T. T. Y., Nor, M. I., Ahmed, E., \& Yahya, S. (2018). The determination of firm performance in emerging nations: Do board size and firm size matter. Management, 5(3), 57-66. DOI 10.9756/IAJAFM/V5I2/1810017

[3] Al-Matari, E. M., Al-Swidi, A. K., \& Bt Fadzil, F. H. (2014). The Measurements of Firm Perfomance's Dimensions. Asian Journal of Finance \& Accounting, 6(1), 24-49. Recuperado el 15 de Marzo de 2020, de https://doi.org/10.5296/ajfa.v6i1.4761

[4] Arosa, B., Iturralde, T., \& Maseda, A. (2010). Outsiders on the board of directors and firm performance: Evidence from Spanish non-listed family firms. Journal of Family Business Strategy, 1(4), 236-245. DOI:10.1016/j.jfbs.2010.10.004

[5] Arosa, B., Iturralde, T., \& Maseda, A. (2013). The board structure and firm performance in SMEs: Evidence from Spain. Investigaciones Europeas de Dirección y Economía de la Empresa, 19(3), 127-135. https://doi.org/10.1016/j.iedee.2012.12.003

[6] Avkiran, N., \& Ringle, C. (2018). Partial Least Squares Structural Equation Modeling: Recent Advances in Banking and Finance. https://doi.org/10.1007/978-3-319-71691-6 
[7] Ayda, F., Normala Obaid, S., \& Azlan, H. (2017). Corporate governance effect on firms' performance evidence from the UAE. Journal of Economic and Administrative Sciences, 33(1), 66-80. https://doi.org/10.1108/JEAS-01-2016-0002

[8] Autor 1- Anonimizado conforme a lineamientos de revista.

[9] Baker, H. K., Pandey, N., Kumar, S., \& Haldar, A. (2020). A bibliometric analysis of board diversity: Current status, development, and future research directions. Journal of Business Research, 108, 232246. https://doi.org/10.1016/j.jbusres.2019.11.025

[10] Barnhart, S. W., Marr, M. W., \& Rosenstein, S. (1994). Firm performance and board composition: Some new evidence. Managerial and decision economics, 15(4), 329-340. https://doi.org/10.1002/mde.4090150407

[11] Black, B. (June de 2001). The Corporate Governance Behavior and Market Value of Russian Firms. $\begin{array}{llll}\text { Emerging } \quad \text { Markets } & \text { Review, } & \text { 89-108. }\end{array}$ https://EconPapers.repec.org/RePEc:eee:ememar:v:2:y:2001:i:2:p:89-108

[12] Black, B. S., Love, I., \& Rachinsky, A. (2005). Corporate Governance and Firm's Market Values. Time Series Evidence from Russia. Emergin Markets Review. DOI:10.1016/j.ememar.2006.09.004

[13] Bagozzi, R. P. (1981). A Prospectus for Theory Construction in Marketing. Journal of Marketing, 48(1), 11-29. https://doi.org/10.2307/1251307

[14] Bermig, A., \& Frick, B. (2010). Board size, board composition, and firm performance: Empirical evidence from Germany. Board Composition, and Firm Performance: Empirical Evidence from Germany June 10, 2010). https://www.researchgate.net/profile/Bernd-Frick2/publication/228293328_Board_Size_Board_Composition_and_Firm_Performance_Empirical_Evide nce_from_Germany/links/5ccc5a4392851c4eab80f0e2/Board-Size-Board-Composition-and-FirmPerformance-Empirical-Evidence-from-Germany.pdf

[15] Besley, S., \& Brigham, E. (2009). Fundamentos de Administración Financiera. México : Cengage .

[16] Bhagat, S., \& Bolton, B. (2008). Corporate governance and firm performance. Journal of Corporate Finance, 14, 257-273. https://doi.org/10.1016/j.jcorpfin.2008.03.006

[17] Brown, L. D., \& Caylor, M. L. (2004). Corporate governance and firm performance. Available at SSRN 586423.

[18] CCE. (2018a). Código de Principios y Mejores Prácticas de Gobierno Corporativo.

[19] CCE. (2018b). Cuestionario para evaluar el grado de adhesión al código de principios y mejores prácticas de Gobierno Corporativo.

[20] Cheah, J. H., Sarstedt, M., Ringle, C. M., Ramayah, T., \& Ting, H. (2018). Convergent Validity Assessment of Formatively Measured Constructs in PLS-SEM: On Using Single-item versus Multi-item Measures in Redundancy Analysis. International Journal of Contemporary Hospitality Management, 30(11), 31923210. ISSN: 0959-6119

[21] Cheng, S. (2008). Board size and the variability of corporate perfomance. Journal of Financial Economics, 87(1), 157-176. https://EconPapers.repec.org/RePEc:eee:jfinec:v:87:y:2008:i:1:p:157176

[22] Chong, \& López de Silanes. (2007). Corporate Governance in Latin America. (U. o. Amsterdam, Ed.) International Development Bank. DOI:10.2139/ssrn.1820067

[23] Chong, A., \& López-de-Silanes, F. (2007). Corporate governance and firm value in Mexico. Investor protection and corporate governance: Firm-level evidence across Latin America, 397-481. http://dx.doi.org/10.1596/978-0-8213-6913-5

[24] Consejo Coordinador Empresarial. (2019). Codigo de Mejores Prácticas de Gobierno Corporativo. Recuperado el Diciembre de 2019, de https://www.cce.org.mx/codigo-de-principios-y-mejorespracticas-de-gobierno-corporativo/ 
[25] Dal Vesco, D. G., \& Beuren, I. M. (2016). Do the Board of Directors Composition and the Board Interlocking Influence on Performance? Brazilian Administration Review, 13(2), 1-26. DOI:10.1590/1807-7692bar2016160007

[26] Daines, R. (2001). Does Delaware law improve firm value? Journal of Financial Economics, 62, 525-558. https://doi.org/10.1016/S0304-405X(01)00086-1

[27] Dasilas, A., \& Papasyriopulos, N. (2015). Corporate governance, credit ratings and the capital structure of Greek SME and large listed firms. Small Business Economics (45), 215-244. https://doi.org/10.1007/s11187-015-9648-y

[28] Demuner , M., \& López , N. (2017). Valuación de Hoteles City Express mediante el método de flujos de efectivo descontados. El Periplo Sustentable, Julio - Diciembre (33). ISSN 1870-9036. Disponible en: $<$ https://rperiplo.uaemex.mx/article/view/4851>

[29] Dey, A. (2008). Governance and Agency Conflicts. Journal of Accounting Research, 46(5), 1143-1181. https://doi.org/10.1111/j.1475-679X.2008.00301.x

[30] Di Pietra, R., Grambovas, C. A., Raonic, I., \& Riccaboni, A. (2008). The effects of board size and "busy" directors on the market value of Italian companies. J Manage Governance, 12. doi:10.1007/10997-0089044-y

[31] Eisenhardt, K. M. (January de 1989). Agency Theory: An Assessment and Review. The Academy of Management Review, 14(1), 57-74. DOI:10.5465/AMR.1989.4279003

[32] Fama, E. F., \& French, K. R. (1993). Common risk factors in the return on stocks \& bonds. Journal of Financial Economics, 33(1), 3-56. https://doi.org/10.1016/0304-405X(93)90023-5

[33] Fama, E. F., \& French, K. R. (2105). A five-factor asset pricing model. Journal of Financial Economics, 116(1), 1-22. https://doi.org/10.1016/j.jfineco.2014.10.010

[34] Farhan, A., Normala Obaid, S., \& Azlan, H. (2017). Corporate governance effect on firms performanceevidence from UAE. Journal of Economics and Administrative Sciences, 33(1), 66-80. doi:https://doi.org/10.1108/JEAS-01-2016-0002

[35] Frick, B., \& Bermig, A. (2009). Board size, board composition and firm performance: Empirical evidence from Germany. Retrieved from https://www. researchgate. net/publication/228293328_Board_Size_Board_Composition_and_Firm_Performance_Empirical_Eviden ce_from_Germany. DOI:10.2139/ssrn.1623103

[36] Fulghieri, P., \& Suominen, M. (2012). Corporate Governance, Finance, and the Real Sector. 47(6), 11871214. DOI: $10.1017 / \mathrm{S} 0022109012000531$

[37] Fuzi, S. F. S., Halim, S. A. A., \& Julizaerma, M. (2016). Board independence and firm performance. Procedia Economics and Finance, 37, 460-465. DOI: $10.1016 / \mathrm{S} 2212-5671(16) 30152-6$

[38] Garg, A. K. (2007). Influence of board size and independence on firm performance: A study of Indian companies. Vikalpa, 32(3), 39-60. DOI:10.1177/0256090920070304

[39] Guest, P. M. (2009). The Impact of Board Size on Firm Perfomance: Evidence from the UK. The European Journal of Finance, 15(4), 385-404. Available at SSRN: https://ssrn.com/abstract=2118221

[40] Gompers, P. A., Ishii, J. L., \& Metrick, A. (2003). Coporate Governance and Equity Prices. Quarterly Journal of Economics, 118(1), 10155. http://dx.doi.org/10.2139/ssrn.278920

[41] Hair, J. F., Hult, G. T., Ringle, C. M., \& Saerstedt, M. (2014). A Primer on Partial Least Squares Structural Equation Modeling (PLS-SEM). SAGE: Thousand Oaks.

[42] Hair, J. F., Risher, J. J., Sarstedt, M., \& Ringle, C. M. (2019). When to Use and How to Report the Results of PLS-SEM. European Business Review, 31(1), 2-24. DOI:10.1108/EBR-11-2018-0203

[43] Henseler, J., Dijkstra, T. K., Sarstedt, M., Ringle, C. M., Diamantopoulos, A., Straub, D. W., \& Calantone, R. J. (2013). Common Beliefs and Reality About PLS: Comments on Rönkkö and Evermann. Organizational Research Methods, 17(2), 182-209. https://doi.org/10.1177/1094428114526928 
REMEF (The Mexican Journal of Economics and Finance)

El desempeño financiero de la empresa y la composición del consejo de administración

[44] Hernandez Chavez, F. A., \& Berrocal Gonzalez, A. M. (2009). El Gobierno Corporativo y la Valuación de la Empresa. ¿Existe relación entre ambos? En I. M. Finanzas, Hacia una Empresa más profesional e institucional. Gobierno Corporativo en México. (págs. 153-175). México.

[45] Hessels, J., \& Terjesen, S. (2010). Resource Dependency and Institutional Theory Perspectives on Direct and Indirect Export Choices. Small Business Economics(2), 203-220. DOI: 10.1007/s11187-008-91564

[46] Hillman, A., Withers, M., \& Colllins, B. (2009). Resource Dependence Theory: A review. Journal of Management Online First. https://doi.org/10.1177/0149206309343469

[47] Ionescu, G. G., \& Negrusa, A. L. (December de 2007). The Study about Organizational Life Cycle Models. Review of International Comparative Management, 8(4), 5-17. Obtenido de http://www.rmci.ase.ro/Login/no8vol4/Vol8_No4_Article1.pdf

[48] Jensen, M. C. (2010). The Modern Industrial Revolution, Exit and the Failure of Iternal Control Systems. Journal of Applied Corporate Finance, 22(1), 43-58. doi:10.1111/j.1745-6622.2010.00260.x.

[49] Jensen, M., \& Mekling, W. (1976). Theory of the Firm:Managerial Behavior, Agency Costs and Ownership Structure. Journal of Financial Economics, 3(4), 305-360. https://doi.org/10.1016/0304405X(76)90026-X

[50] Kabir, R., \& Minh Thai, H. (2017). "Does corporate governace shape the relationship between corporate social responsability and financial performance?". Pacific Accounting Review, 29(2), 227-258. doi:http://doi.org/10.1108/PAR-10-2016-0091

[51] Kang, S.-A., \& Kim, Y.-S. (2012). Effect of Corporate Governance on Real Activity-Based Earning Management: Evidence from Korea. Journal of Business Economics and Management, 13(1), 29-52. DOI:10.3846/16111699.2011.620164

[52] Kao, M.-F., Hodgkinson, L., \& Jaafar, A. (2019). Ownership structure, board of directors and firm performance: evidence from Taiwan. Corporate Governance: The International Journal of Business in Society. DOI:10.1108/CG-04-2018-0144

[53] Kent Baker, H., Pandey, N., Kumar, S., \& Haldar, A. (2020). A bibliometric analysis of board diversity: Current status, development, and future research directions. Journal of Business Research, 108, 232246. doi:https://doi.org/10.1016/j.jbusres.2019.11.025

[54] Kim, Y. (2005). Board network characteristics and firm performance in Korea. Corporate Governance: An International Review, 13(6), 800-808. https://doi.org/10.1111/j.1467-8683.2005.00471.x

[55] Klein, A. (1998). Firm performance and board committee structure. The Journal of Law and Economics, 41(1), 275-304. http://dx.doi.org/10.1086/467391

[56] La Porta, R., Lopez de Silanes, F., Shleifer, A., \& Vishny, R. (January de 2000). Investor Protection and Corporate Governance. Journal of Financial Economics, 58(1-2), 3-27. Recuperado de: https://scholar.harvard.edu/files/shleifer/files/ip_corpgov.pdf

[57] Lebas, M., \& Euske, K. (2002). A conceptual and operational delineation of performance. En A. Neely, Business Performance Measurement: Theory and Practice (págs. 65-79). Cambridge, United Kingdom: Cambridge University Press. ISBN 0-511-04157-8 eBook Cambridge University Press 20042002 (netLibrary)

[58] Li, J., Nan, L., \& Zhao, R. (2018). Corporate governance roles of information quality and corporate take overs. Account Studies . DOI:10.1007/s11142-018-9449-z.

[59] Lintner, J. (1965). The Valuation of Risk Assets and the Selection of Risky Investments in Stock Portfolios and Capital Budgets. The Review of Economics and Statistics, 1, 13-37. https://doi.org/10.2307/1924119

[60] Liu, Y., Miletkov, M. K., Wei, Z., \& Yang, T. (2015). Board independence and firm performance in China. Journal of corporate finance, 30, 223-244. DOI: 10.1016/j.jcorpfin.2014.12.004

[61] Lopez de Prado, M. (2018). Advances in Financial Machine Learning. Wiley. 
[62] Louma, P., \& Goodstein, J. (1999). Stakeholders and Corporate Boards: Institutional Influences on Board Composition and Structure. The Academy of Maanagement Journal, 42(5), 553-563. https://doi.org/10.2307/256976

[63] Macías, A. J., \& Román, F. J. (2014). Consecuencias económicas de la Reforma de Gobierno Corporativo en un mercado de capitales emergente. Pruebas de México. El trimestre económico, 81(322), 357-412. Retrieved from SCIELO: S2448-718X2014000200357 DOI https://doi.org/10.20430/ete.v81i322.117

[64] Marine, F. (2019). Tesis Doctoral. Análisis y evaluación de la conducta del mercado mexicano de valores, de sus inversionistas y de sus emisoras más relevantes por medio del uso de constructos, bursátiles, económicos $e$ internacionales. México: Universidad Anáhuac. https://anahuac.primo.exlibrisgroup.com/permalink/52ANAHUAC_INST/148j1ej/alma9938672796 05016

[65] Martínez Ávila, M., \& Fierro Moreno, E. (2018). Aplicación de la técnica PLS-SEM en la gestión del conocimiento: un enfoque técnico práctico. RIDE. Revista Iberoamericana para la Investigación y el Desarrollo Educativo, 8(16), 130-164. https://doi.org/10.23913/ride.v8i16.336

[66] Neely, A., Mike, G., \& Platts, K. (1995). Peformance measurement system design: A literature review and research agenda. International Journal of Operation \& Production Management, 15(5), 80-116. Recuperado el Marzo de 2020, de https://doi.org/10.1108/01443579510083622

[67] OECD. (2016). Principios de Gobierno Corporativo de la OCDE y del G20. Retrieved from https://www.oecd.org/daf/ca/corporategovernanceprinciples/37191543.pdf http://dx.doi.org/10.1787/9789264259171-es

[68] Orozco, L. A., Vargas, J., \& Galindo-Dorado, R. (2018). Trends on the relationship between board siza and financial and reputational corporate performace: The Colombian case. European Journal of Management and Business Economics, 27(2), 183-197. doi: http://doi.org/10.1108/EJMBE-02-20180029

[69] Perrow, C. (1993). Complex organizations: a criticial essay. (3rd. ed) McGraw-Hill- New York.

[70] Pfeffer, J., \& Salancik, G. R. (1978). The External Control of Organizations. A Resource Dependence Perspective. New York: Harper \& Row.

[71] Pfeffer, J., \& Salancik, G. R. (2003). The External Control of Organizations. A Resource Dependence Perspective. Stanford, California, USA: Standford University Press. Recuperado el 30 de Septiembre de 2018

[72] La Porta, R., Lopez-de-Silanes, F., Shleifer, A., \& Vishny, R. W. (1999). Investor protection: origins, $\begin{array}{lll}\text { consequences, } & \text { reform. http://www. }\end{array}$ https://www.nber.org/system/files/working_papers/w7428/w7428.pdf

[73] Rashid, A. (2018). Board independence and firm performance: Evidence from Bangladesh. Future Business Journal, 4(1), 34-49. https://doi.org/10.1016/j.fbj.2017.11.003

[74] Reinartz, W. J., Haenlein, M., \& Henseler, J. (2009). An empirical comparison of the efficacy of covariance-based and variance-based SEM. International Journal of Research in Marketing, 332-344. DOI: 10.1016/j.ijresmar.2009.08.001

[75] Reyna, J. M. S. M. (2012). Interacción entre Mecanismos Internos y Externos de Gobierno como Elemento Detonante de la Creación de Valor: un estudio internacional. Revista Brasileira de Gestão de Negócios, 14 (43), 143-158. ISSN1806-4892

[76] Ross, S. (1976). The arbitrage theory of capital asset pricing. Journal of Economic Theory, 13(3), 341360. https://doi.org/10.1016/0022-0531(76)90046-6

[77] Saif Ullah, M. (2012). Relationship between Corporate Governace Score and Stock Prices: Evidence from KSE-30 Index Companies. Journal of Business and Social Science, 3(4), 239-249. Available at SSRN: https://ssrn.com/abstract=1989875 
REMEF (The Mexican Journal of Economics and Finance)

El desempeño financiero de la empresa y la composición del consejo de administración

[78] Sharpe, W. F. (1964). Capital asset pricecs: A theory of market equilibrum under conditions of risk. Journal of Finance, 427-442. https://doi.org/10.2307/2977928

[79] Song, H. J., Yoon, Y. N., \& Kang, K. H. (2020). The relationship between board diversity and firm performance in the lodging industry: The moderating role of internationalization. International Journal of Hospitality Management, 86, 102461. doi: https://doi.org/10.1016/j.ijhm.2020.102461

[80] Tejedo-Romero, F., \& Ferraz, J. (2018). Estrategia de gestión y divulgación del capital intelectual: influencia del gobierno corporativo. Contaduría y Administración, 2(63), 1-18. Obtenido de www.cya.unam.mx/index.php/cya

DOI: http://dx.doi.org/10.22201/fca.24488410e.2018.1236

[81] Thaler, R. (2016). Portarse mal. El comportamiento irracional en la vida económica. Paidós.

[82] Topak, M. S. (2011). The effect of board size on firm performance: Evidence from Turkey. Middle Eastern Finance and Economics, 14(1), ISSN:1450-2889.

[83] Tosun, 0. K. (2020). Changes in corporate governance: Externally dictated vs voluntarily determined. International Review of Financial Analysis, 101608. doi: https://doi.org/10.1016/j.irfa.2020.101608

[84] Tricker, B. (2012). Corporate Governance. Principles, Polices, and Practices. Oxford, United Kingdom: Oxford, University Press.

[85] Ullah, S., Ahmad, S., Akbar, S., Kodwani, D., \& Frecknall-Hughes, J. (2020). Governance disclosure quality and market valuation of firms inUKand Germany. International Journal of Finance \& Economics. doi:10.1002/ijfe.2053

[86] Uribe-Bohorquez, M.-V., Martínez-Ferrero, J., \& García-Sánchez, I.-M. (2018). Board independence and firm performance: The moderating effect of institutional context. Journal of Business Research, 88, 2843. doi: https://doi.org/10.1016/j.jbusres.2018.03.005

[87] Valencia Herrera, H., \& Ruiz Rivera, F. J. (2019). Dividend payout policy, family control, and institutional investors in an emerging country: The case of Mexico. Contaduría y administración, 64(4). https://doi.org/10.22201/fca.24488410e.2019.1762

[88] Vargas Chanes, D. (2019). Aspectos metodológicos para la investigación social: Modelos de ecuaciones estructurales. Ciudad de México: Universidad Autónoma de México.

[89] Wang, Y.-C., Tsai, J.-J., \& Willian, H.-W. L. (Junio de 2013). The Influence of Board Structure on Firm Perfomance. The Journal of Global Business Management, 9(2), 7-14. Recuperado de: http://www.jgbm.org/page/2\%20Yang-Chao\%20Wang-1.pdf

[90] Watkins Fassler, K. (2018). Desempeño financiero de empresas familiares. (F. d. Administración, Ed.) Contaduría y Administración, 2(63), 1-18. Obtenido de www.cya.unam.mx/index.php/cya ISSN: 01861042. https://doi.org/10.22201/fca.24488410e.2018.1214

[91] Yermack, D. (1996). Higher market valuation of companies with a small board of directors. Journal of Financial Economics, 40(2), 185-211. https://doi.org/10.1016/0304-405X(95)00844-5

[92] Zabri, S. M., Ahmad, K., \& Wah, K. K. (2016). Corporate governance practices and firm performance: Evidence from top 100 public listed companies in Malaysia. Procedia Economics and Finance, 35, 287296. doi:10.1016/S2212-5671(16)00036-8

[93] Zhou, H., Owusu-Ansah, S., \& Maggina, A. (2018). Board of directors, audit committee, and firm performance: Evidence from Greece. Journal of International Accounting, Auditing and Taxation, 31, 2036. DOI: $10.1016 /$ j.intaccaudtax.2018.03.002

[94] Wang, Y.-C., Tsai, J.-J., \& Willian, H.-W. L. (Junio de 2013). The Influence of Board Structure on Firm Perfomance. The Journal of Global Business Management, 9(2), 7-14.

[95] Yermack, D. (1996). Higher market valuation of companies with a small board of directors. Journal of Financial Economics, 40(2), 185-211. 
[96] Zabri, S. M., Ahmad, K., \& Wah, K. K. (2016). Corporate governance practices and firm performance: Evidence from top 100 public listed companies in Malaysia. Procedia Economics and Finance, 35, 287296.

[97] Zhou, H., Owusu-Ansah, S., \& Maggina, A. (2018). Board of directors, audit committee, and firm performance: Evidence from Greece. Journal of International Accounting, Auditing and Taxation, 31, 20 36.

\section{Anexos}

1.-Path analysis modelo general

\begin{tabular}{|l|c|c|c|}
\hline & RETORNO & $\begin{array}{c}\text { REVENUE PER } \\
\text { EMPLOYEE }\end{array}$ & $\begin{array}{c}\text { RIESGO } \\
\text { SISTEMÁTICO }\end{array}$ \\
\hline $\begin{array}{l}\text { CONSEJEROS } \\
\text { INDEPENDIENTES }\end{array}$ & -0.587 & -0.203 & 0.634 \\
\hline $\begin{array}{l}\text { CONSEJEROS } \\
\text { PATRIMONIALES }\end{array}$ & -0.348 & -0.374 & 0.119 \\
\hline CONSEJEROS RELACIONADOS & -0.328 & -0.46 & 0.178 \\
\hline
\end{tabular}

2.- Modelo 2 Convergence Validity.

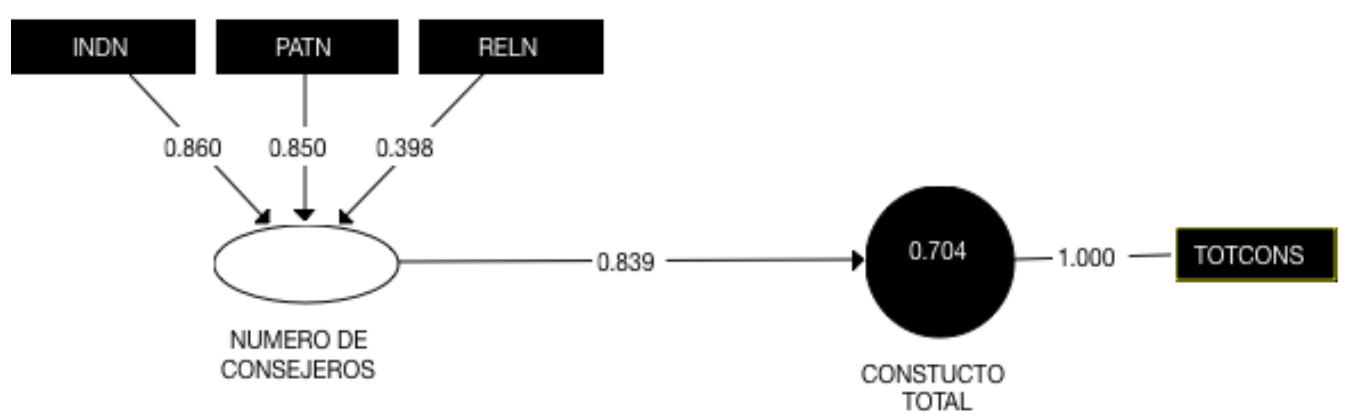

3.-Rsquare

\begin{tabular}{|l|c|c|}
\hline & R Square & R Square Adjusted \\
\hline REVENUE PER EMPLOYEE & 0.245 & 0.161 \\
\hline RETORNO & 0.372 & 0.302 \\
\hline RIESGO SISTEMATICO & 0.377 & 3.000 \\
\hline
\end{tabular}


4.-Fsquare

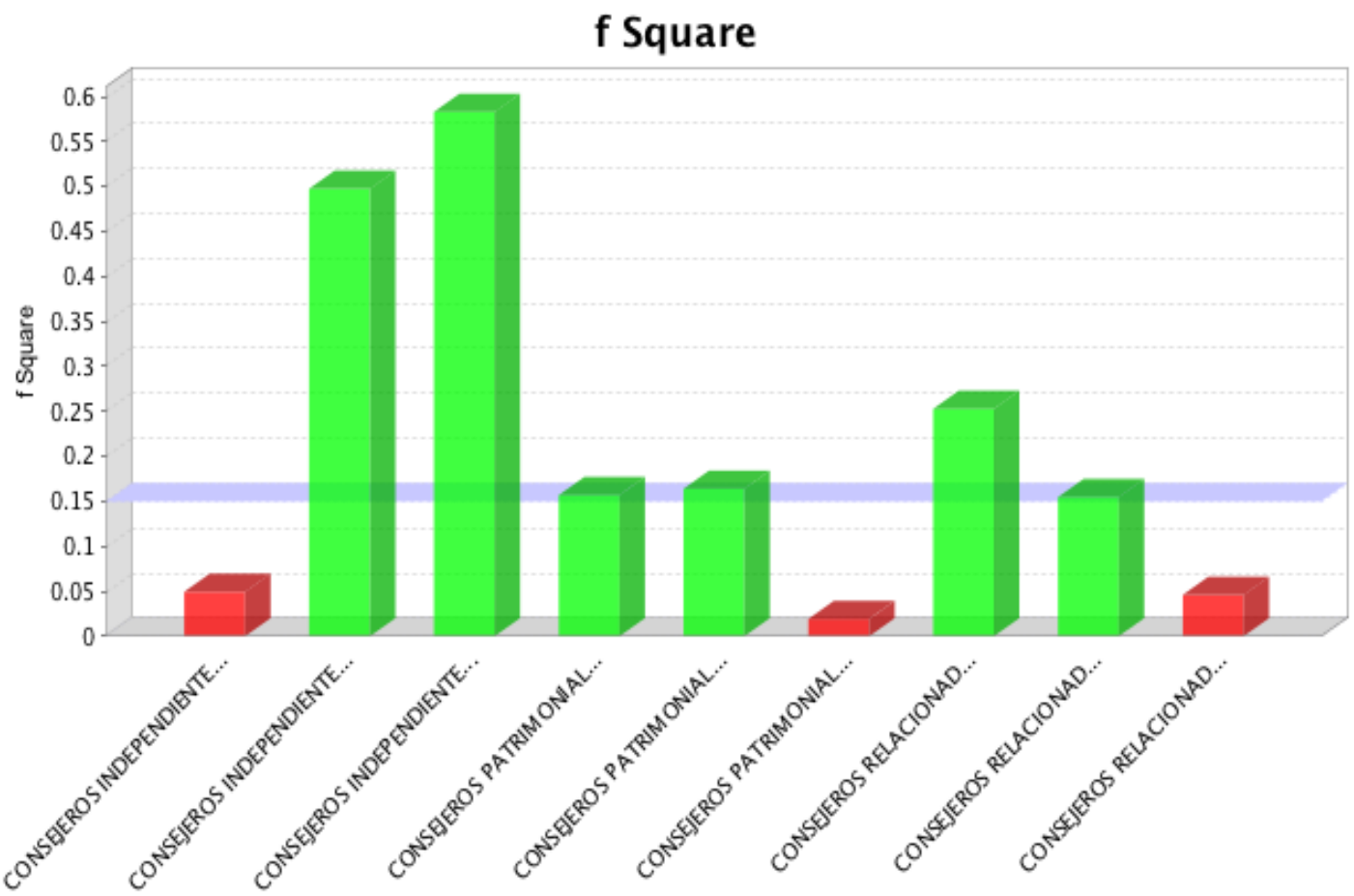

5.-Validity y Reliability

\begin{tabular}{|l|c|c|c|c|}
\hline & $\begin{array}{c}\text { Cronbach's } \\
\text { Alpha }\end{array}$ & rho_A & $\begin{array}{c}\text { Composite } \\
\text { Reliability }\end{array}$ & AVE \\
\hline $\begin{array}{l}\text { CONSEJEROS } \\
\text { INDEPENDIENTES_ }\end{array}$ & 1.000 & 1.000 & 1.000 & 1.000 \\
\hline CONSEJEROS PATRIMONIALES & 1.000 & 1.000 & 1.000 & 1.000 \\
\hline CONSEJEROS RELACIONADOS & 1.000 & 1.000 & 1.000 & 1.000 \\
\hline $\begin{array}{l}\text { REVENUE PER } \\
\text { EMPLOYEEREVENUE PER } \\
\text { EMPLOYEE }\end{array}$ & 1.000 & 1.000 & 1.000 & 1.000 \\
\hline RETORNO & 0.515 & 0.568 & 0.761 & 0.525 \\
\hline RIESGO SISTEMATICO & 1.000 & 1.000 & 1.000 & 1.000 \\
\hline
\end{tabular}

6.-Fornell Larcker Criteria

\begin{tabular}{|c|c|c|c|c|c|c|}
\hline & CONSEJEROS INDEPENDIENTES & CONSEJEROS PATRIMONIALES & CONSEJEROS RELACIONADOS & EFICIENCIA POR EMP & RETORNO & RIESGO SISTEMATICO \\
\hline CONSEJEROS INDEPENDIENTES & 1.000 & & & & & \\
\hline CONSEJEROS PATRIMONIALES & -0.260 & 1.000 & & & & \\
\hline CONSEJEROS RELACIONADOS & -0.091 & -0.264 & 1.000 & & & \\
\hline EFICIENCIA POR EMPLEADO & -0.064 & -0.200 & -0.343 & 1.000 & & \\
\hline RETORNO & -0.467 & -0.109 & -0.183 & 0.434 & 0.725 & \\
\hline RIESGO SISTEMATICO & 0.587 & -0.093 & 0.089 & -0.223 & -0.195 & 1.000 \\
\hline
\end{tabular}


7.-HTMT (heterotrait-monotrait ratio of correlations)

\begin{tabular}{|l|r|r|r|r|r|r|}
\hline & CONSEJEROS INDEPENDIENTES & CONSEJEROS PATRIMONIALES & CONSEJEROS RELACIONADOS & EFICIENCIA POR EMPLEADO & RETORNO & RIESGO SISTEMATICO \\
\hline CONSEJEROS INDEPENDIENTES_ & & & & \\
\hline CONSEJEROS PATRIMONIALES & 0.260 & & & \\
\hline CONSEJEROS RELACIONADOS & 0.091 & 0.264 & & \\
\hline EFICIENCIA POR EMPLEADO & 0.064 & 0.200 & & \\
\hline RETORNO & 0.631 & 0.142 & & \\
\hline RIESGO SISTEMATICO & 0.587 & 0.093 & & & \\
\hline
\end{tabular}

8.-VIF

\begin{tabular}{|l|r|}
\hline & VIF \\
\hline BETA & 1.000 \\
\hline INDN & 1.000 \\
\hline PATN & 1.000 \\
\hline RELN & 1.000 \\
\hline REVENUE PER EMPLOYEE_ & 1.000 \\
\hline ROA_ & 1.625 \\
\hline ROE_ & 1.521 \\
\hline ROS_ & 1.120 \\
\hline
\end{tabular}

9.- VIF (2)

\begin{tabular}{|c|c|c|c|c|c|c|}
\hline & CONSEJEROS INDEPENDIENTES & CONSEJEROS PATRIMONIALES & CONSEJEROS RELACIONADOS & EFICIENCIA POR EMPLEADO & RETORNO & RIESGO SISTEMATICO \\
\hline CONSEJEROS INDEPENDIENTES & & & & 1.105 & 1.105 & 1.105 \\
\hline CONSEJEROS PATRIMONIALES & & & & 1.178 & 1.178 & 1.178 \\
\hline CONSEJEROS RELACIONADOS & & & & 1.107 & 1.107 & 1.107 \\
\hline \multicolumn{7}{|l|}{ EFICIENCIA POR EMPLEADO } \\
\hline RETORNO & & & & & & \\
\hline RIESGO SISTEMATICO & & & & & & \\
\hline
\end{tabular}

10.-Model Fit

\begin{tabular}{|l|c|c|}
\hline & Saturated Model & Estimated Model \\
\hline SRMR & 0.101 & 0.118 \\
\hline d_ULS & 0.366 & 0.502 \\
\hline d_G1 & 0.088 & 0.156 \\
\hline d_G2 & 0.072 & 0.127 \\
\hline Chi-Square & 12.354 & 19.728 \\
\hline NFI & 0.817 & 0.708 \\
\hline
\end{tabular}


11.-Crossvalidated Redundancy (kfold=3)

\begin{tabular}{|l|c|c|c|}
\hline & SSO & SSE & $\begin{array}{c}\mathbf{Q}^{\mathbf{2}} \mathbf{( = 1 -} \\
\text { SSE/SSO })\end{array}$ \\
\hline CONSEJEROS INDEPENDIENTES & 31.000 & 31.000 & \\
\hline CONSEJEROS PATRIMONIALES & 31.000 & 31.000 & \\
\hline CONSEJEROS RELACIONADOS & 31.000 & 31.000 & 0.191 \\
\hline $\begin{array}{l}\text { REVENUE PER } \\
\text { EMPLOYEEREVENUE PER } \\
\text { EMPLOYEE }\end{array}$ & 31.000 & 25.091 & 0.148 \\
\hline RETORNO & 93.000 & 79.224 & 0.119 \\
\hline RIESGO SISTEMATICO & 31.000 & 27.321 & \\
\hline
\end{tabular}

12.-Diferencia entre el modelo estimado por PLS y por LM

PLS

LM

PLS-LM

\begin{tabular}{|l|c|c|c|c|c|r|r|r|r|r|r|l|}
\hline & RMSE & MAE & MAPE & Q2 & RMSE & MAE & MAPE & Q2 & RMSE & MAE & MAPE & Q2 \\
\hline $\begin{array}{l}\text { REVENUE } \\
\text { PER } \\
\text { EMPLOYEE }\end{array}$ & 4.96 & 3.62 & 259.40 & 0.05 & 4.96 & 3.62 & 259.40 & 0.05 & & & & \\
\hline ROA_ & 0.04 & 0.03 & 60.48 & 0.17 & 0.04 & 0.03 & 62.09 & 0.15 & 0.00 & 0.00 & -1.61 & 0.02 \\
\hline ROE_ & 0.13 & 0.09 & 61.79 & 0.03 & 0.14 & 0.09 & 65.95 & -0.01 & 0.00 & 0.00 & -4.17 & 0.05 \\
\hline $\begin{array}{l}\text { MARGEN } \\
\text { DE } \\
\begin{array}{l}\text { BENEFICIO } \\
\text { NETO_ }\end{array}\end{array}$ & 12.97 & 10.40 & 191.81 & 0.00 & 13.47 & 10.85 & 183.10 & -0.07 & & & & \\
\hline BETA & 0.24 & 0.20 & 27.05 & 0.24 & 0.24 & 0.20 & 27.05 & 0.24 & 0.00 & 0.00 & 0.00 & 0.00 \\
\hline
\end{tabular}

13.-Factor de Inflación de la Varianza (VIF)

\begin{tabular}{|l|c|}
\hline & VIF \\
\hline INDN & $\mathbf{1 . 1 0 5}$ \\
\hline PATN & $\mathbf{1 . 1 7 8}$ \\
\hline RELN & $\mathbf{1 . 1 0 7}$ \\
\hline TOTCONS & $\mathbf{1 . 0 0 0}$ \\
\hline
\end{tabular}

